\title{
Caracterización de efectos sísmicos locales en la ciudad de Lorca
}

\author{
SARa Figueras Vila ${ }^{1}$, Albert Macau Roig ${ }^{1}$, Myriam BelvauX ${ }^{2}$, MiQuel \\ PEIX TARRES ${ }^{1}$, BEATRIZ BENJUMEA MORENO ${ }^{1}$, ANNA GABÀs GASA ${ }^{1}$, TERESA \\ SUSAGNA VIDAL ${ }^{1} \&$ XAVIER GOULA SURIÑACH ${ }^{1}$ \\ ${ }^{1}$ Institut Geològic de Catalunya, c/ Balmes 209-211, 08006, Barcelona. \\ ${ }^{2}$ BRGM, 3, Avenue C. Guillemin, BP 36009, 45060 Cedex 2, Orleans, Francia. \\ sfigueras@igc.cat
}

Recibido: $16 / 05 / 2012$

Aceptado: 18/10/2012

\section{Resumen}

Este estudio está enfocado a la caracterización de efectos sísmicos locales en la ciudad de Lorca y corresponde a uno de los aspectos de interés de una campaña de intervención post-sísmica organizada por Institut Geològic de Catalunya (IGC), la Universitat Politécnica de Catalunya (UPC), la Asociación Española de Ingeniería Sísmica (AEIS) conjuntamente con las asociaciones de ingeniería sísmica francesa (AFPS) y portuguesa (SPES) con el objetivo de calibrar, verificar y validar las metodologías de evaluación del riesgo sísmico que estos organismos utilizan.

Se presenta el trabajo realizado y los resultados obtenidos para caracterizar la capa de suelos blandos aplicando dos técnicas basadas en medidas de ruido sísmico ambiental: la técnica del cociente espectral H/V y la técnica de "array". El registro de réplicas en distintos emplazamientos ha permitido obtener la respuesta sísmica de estos sitios. A partir de la información obtenida en los trabajos geofísicos de campo se han definido columnas de suelo representativas, se ha podido clasificar el tipo de suelo según el Eurocódigo 8, se ha modelizado su respuesta sísmica y se ha estimado el incremento de intensidad macrosísmica debido a la presencia de suelos blandos.

Palabras clave: Efectos locales, ruido sísmico, Vs30, EC8, Simulación numérica, Sismo de Lorca.

\section{Site effects characterization in Lorca city}

\begin{abstract}
This study is focused on the characterization of seismic local effects in Lorca city. This aspect fits to one of the interesting points of a field survey for a post-seismic inspection organized by Institut Geològic of Catalonia (IGC), the Universitat Politècnica of Catalonia (UPC), the Spanish Association of Seismic Engineering (AEIS) together with the Earthquake Engineering Associations of France (AFPS) and Portugal (SPES) with the aim to calibrate, check and validate methodologies for seismic risk assessment that these organisms use.

We present the work done and the obtained results in order to characterize the soil layers applying two passive seismic techniques: the H/V spectral ratio and the array method. The record of aftershocks in different emplacements has allowed us to obtain the soil seismic response of these sites. The information obtained from the geophysical field survey allowed the definition of representative soil columns and the soil classification according the Eurocode 8. Subsequently, the soil seismic response has been modelled and the increase of macroseismic intensity due to the presence of soft soils has been assessed.
\end{abstract}

Keywords: site effects, seismic noise, Vs30, EC8, numerical simulation, Lorca earthquake.

Referencia normalizada

Figueras, S., Macau, A., Peix, M., Benjumea, B., Gabas, A., Susagna, T., \& Goula, X. (2012). Caracterización de efectos sísmicos locales en la ciudad de Lorca. Física de la Tierra, 24, 235-254. 
Sumario: Introducción. 1. Los efectos sísmicos locales. 2. Estudios previos realizados en Lorca. 3. Campaña de intervención post-sísmica en Lorca. 3.1 Obtención de la frecuencia fundamental del suelo. 3.2. Clasificación de suelos según el Eurocódigo-8. 3.3 Red de intervención para el registro de réplicas. 4. Modelización de la respuesta sísmica del suelo. 5. Conclusiones. Agradecimientos. Referencias bibliográficas.

\section{Introducción}

El municipio de Lorca está situado al SW de la región de Murcia, en el sector oriental de la Cordillera Bética que constituye el extremo más occidental del cinturón orogénico Alpino. Esta región está sometida a una compresión global de orientación NNW-SSE relacionada con la convergencia de las placas tectónicas Euroasiática y Africana. A pocos quilómetros del $\mathrm{N}$ de la ciudad de Lorca se produce el contacto entre el dominio de la Zona Interna Bética, definida por el desplazamiento de la corteza y subcorteza, y el dominio de la Zona Externa Bética, definida por la inversión y deformación del margen pasivo mesozoico (IGME, 2011).

La Cuenca de Lorca está delimitada, al Norte por la falla Cádiz-Alicante, de dirección NE-SW, y al Sur por la falla Alhama de Murcia de dirección ENE-SW. Ambos sistemas son los responsables de los mecanismos locales de deformación reciente, desde finales del Mioceno hasta la actualidad.

La estructura de la falla de Alhama de Murcia en la zona de Lorca es bastante compleja, con varias ramas que pasan muy próximas a la ciudad. La actividad de esta falla durante los últimos 2 o 3 millones de años ha producido el levantamiento de sierras al noroeste y el hundimiento del valle al sureste, la ciudad de Lorca está situada en una de las laderas levantadas y se extiende desde la Sierra de la Tercia hasta el valle del rio Guadalentín. La información geológica disponible indica una gran variabilidad de sus características geológicas, (IGN, 2011).

Gran parte de la ciudad se sitúa prácticamente sobre la falla responsable del sismo del día 11 de mayo de 2011, de $\mathrm{Mw}=5.1$. Durante los días posteriores al sismo se constataron diferencias geográficas en la distribución de daños en los edificios. Por un lado se observó que el sector más afectado fue el barrio de la Viña (suelo blando), situado al sur de la ciudad, y por tanto más alejado del epicentro. También se observó que los edificios situados en el llano, construidos sobre suelo blando, habían sufrido más daños que los de la parte alta, construidos sobre terreno firme (roca) independientemente de su edad y de sus características constructivas (IGN, 2011).

Las diferencias en la distribución de daños observadas en Lorca junto con la geología particular de la ciudad, sugieren la presencia de efectos de suelo durante el sismo.

\section{Los efectos sísmicos locales}

El movimiento del suelo durante un terremoto puede ser amplificado por las condiciones geológicas, geotécnicas y topográficas del terreno por el que se 
propagan las ondas sísmicas que provienen del sustrato rocoso. La modificación del movimiento del suelo en su amplitud, duración y contenido frecuencial se conoce con el nombre de efecto sísmico local y juega un papel muy importante en el grado de distribución de daños durante un terremoto (Bard, P.Y., 1995; Aki, K., 1993).

Dentro de los efectos sísmicos locales distinguimos los debidos al suelo y a la topografía (Figueras et al., 2009) y los efectos indirectos como la licuefacción y los deslizamientos de ladera (Jiménez et al., 2009). En este artículo analizaremos los efectos de suelo y utilizaremos indistintamente los términos efecto local y efecto de suelo.

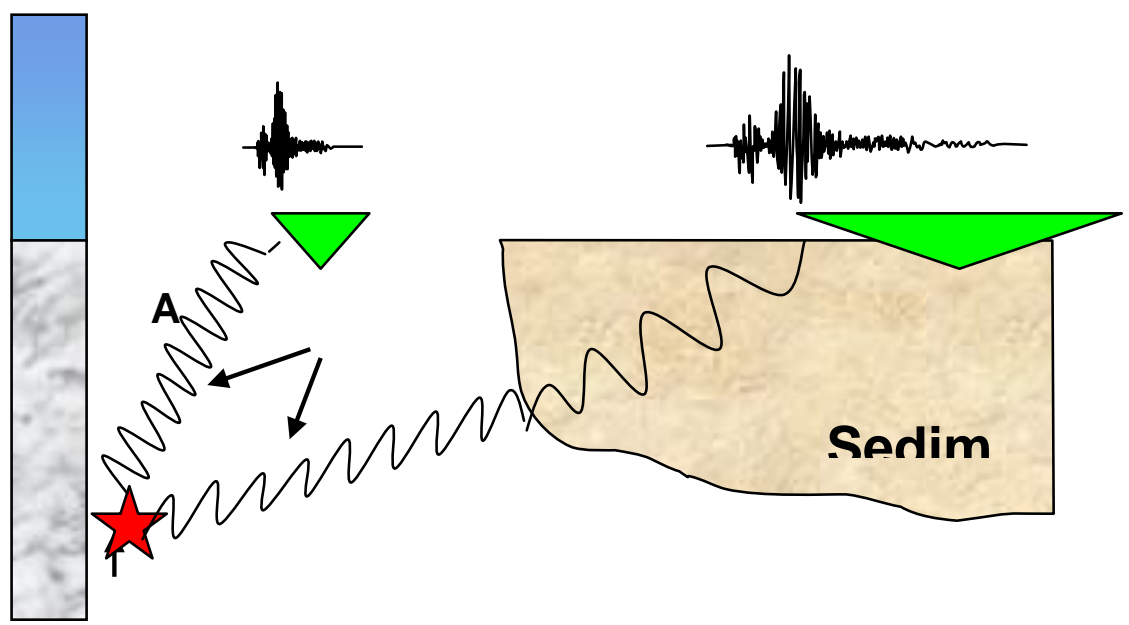

Fig. 1. Registros sísmicos en emplazamientos próximos pero con características del subsuelo distintas. A: roca, B: suelo. Modificación de las ondas sísmicas al propagarse en sedimentos blandos.

En muchos terremotos destructores la geología superficial de un área urbana ha influido notablemente en el nivel de la sacudida sísmica, sobretodo en el rango de periodos que coincide con el periodo de vibración de las estructuras.

Los efectos sísmicos locales tienen que ser caracterizados en los estudios de peligrosidad sísmica a escala local, los resultados de estos estudios se presentan en forma de mapas de microzonificación sísmica y aceleraciones espectrales en distintos tipos de suelo, que serán considerados en la planificación del territorio, en el diseño antisísmico de estructuras y en la revisión de las normas de construcción sismo-resistente.

En Macau (2008), se propone un conjunto de metodologías para la zonación de la peligrosidad sísmica a escala local en las que se consideran diferentes aproximaciones para caracterizar la amplificación del movimiento del suelo con distintos niveles de precisión en función de la información disponible de la zona de estudio y de los parámetros físicos que se pretende analizar. 


\section{Estudios previos realizados en Lorca}

El municipio de Lorca ha sido objeto de estudios previos de peligrosidad y vulnerabilidad sísmica tanto a escala regional (proyecto RISMUR, Benito et al, 2006, DGPC., 2006) como a escala local (ITGE, 1992; Navarro et al. 2006, Navarro et al., 2008, Navarro et al., 2012).

Como resultado de estos estudios se dispone de una clasificación geológica de la ciudad de Lorca así como de una clasificación EC8 del suelo de Lorca (figura 2) basada en valores $\mathrm{VS}_{30}$ obtenidos en campañas geofísicas, (Navarro et al., 2008).

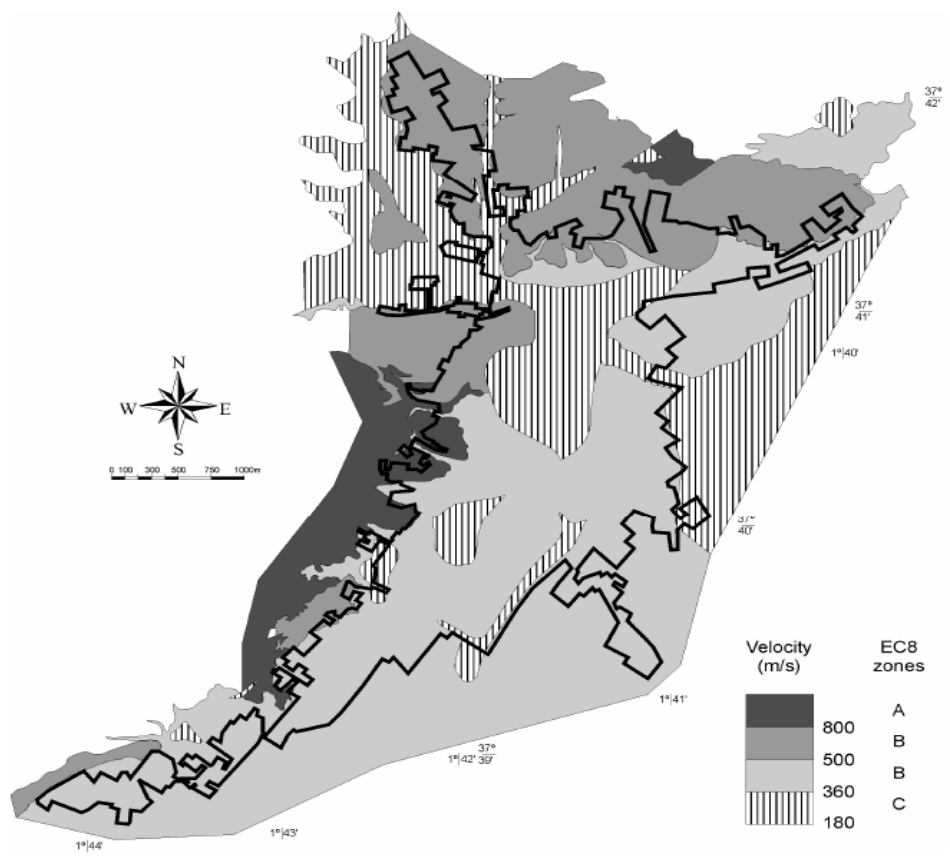

Fig. 2. Mapa de clasificación de suelos de Lorca según el EC-8, basado en los valores de $\mathrm{V}_{\mathrm{S}}{ }^{30}$. El contorno negro representa el límite urbano. (Navarro et al., 2008).

De forma muy resumida se puede decir que la parte Oeste de la ciudad, dónde se ubica el centro histórico situada en los contrafuertes del Castillo, aparecen afloramientos rocosos de edad paleozoica (suelo tipo A), hacia el Noreste predominan materiales Miocénicos (Tortonianos-Margas y conglomerados) de roca media-dura (B), el resto de la ciudad se ubica en suelos formados por materiales cuaternarios del Pleistoceno (Glacis), roca blanda (B) y más al Este, la parte más moderna de la ciudad, está situada sobre materiales coluviales y aluviales recientes (C). El espesor de sedimentos tiende a aumentar hacia la cuenca del rio Guadalentín. La expansión urbanística ha favorecido la ocupación de los suelos blandos del valle del Guadalentín. 
También existe un estudio previo de amplificación de suelos a partir del registro de terremotos de magnitudes entre 1.9 y 4.7 , y profundidades entre 4 y $20 \mathrm{~km}$ en una red local de acelerógrafos instalados en Lorca en emplazamientos con distintas características del subsuelo. En este estudio se observa que los registros obtenidos en estaciones emplazadas en suelo tienen mayores amplitudes que los del emplazamiento de referencia. En las funciones de transferencia se observan amplificaciones con factores entre 5 y 7 en suelos blandos respecto al emplazamiento de referencia para un rango de periodos entre 0,1 y $0,3 \mathrm{~s}$. (Navarro et al. 2006)

\section{Campaña de intervención post-sísmica en Lorca}

Con el objetivo de comprobar los posibles efectos de suelo se realizó una campaña de caracterización de los efectos sísmicos locales en la ciudad de Lorca. Los resultados de los estudios citados en el apartado anterior se tuvieron en cuenta para planificar la campaña de campo.

Los trabajos de campo se llevaron a cabo entre los días 24 y 27 de Mayo de 2011.

Uno de los objetivos era caracterizar la capa de suelos blandos, para ello se obtuvieron registros de ruido sísmico ambiental puntuales, para evaluar las frecuencias fundamentales de los distintos tipos de suelo e intentar correlacionar los eventuales efectos de sitio con los daños observados. Además, se realizaron medidas a lo largo de perfiles perpendiculares al rio Guadalentín para observar la evolución de las relaciones espectrales. También se realizaron registros de ruido sísmico ambiental en array para obtener el perfil de velocidad de las ondas de cizalla $\left(\mathrm{V}_{\mathrm{S}}\right)$ en las capas de suelo.

El segundo objetivo era caracterizar la respuesta sísmica de la capa de suelos, para ello se instalaron sismógrafos en tres emplazamientos distintos para cuantificar los efectos de suelo aplicando el método suelo/referencia con registros de posibles réplicas. Se pudo disponer también de los registros de la unidad móvil del IGC emplazada en el delta del rio Gaudalentín.

En la figura 3 se ubican los sismógrafos de la red de intervención y se muestra la situación de los emplazamientos donde se realizaron las medidas de ruido sísmico y las medidas de ruido en array. 


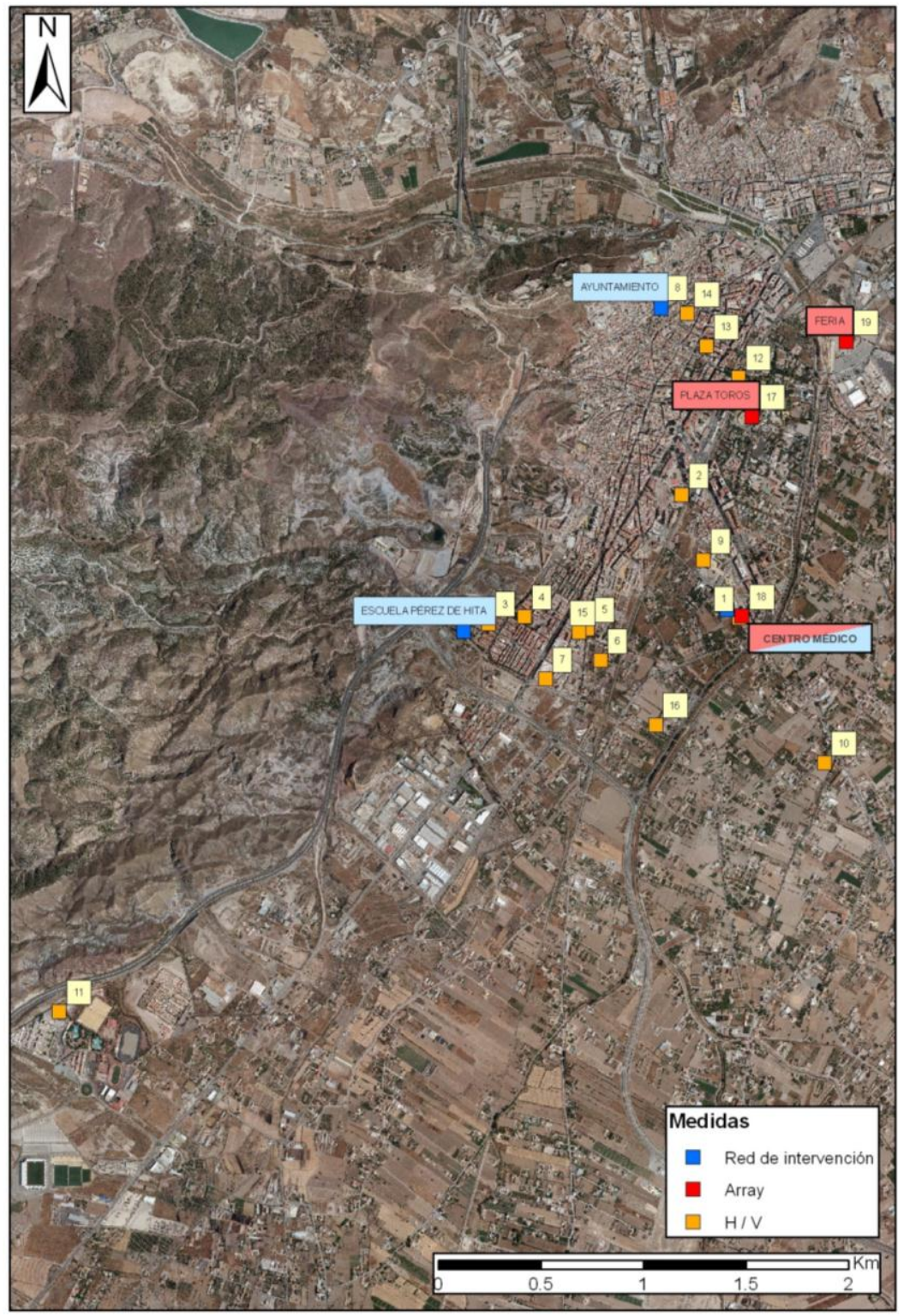

Fig. 3. Situación de las estaciones de la red de intervención y localización de las medidas de ruido sísmico (cociente espectral H/V y técnica de array) realizadas en la ciudad de Lorca.

\subsection{Obtención de la frecuencia fundamental del suelo}

Se midió el ruido sísmico en 19 emplazamientos con el objetivo de aplicar la técnica del cociente espectral H/V y obtener la frecuencia fundamental del suelo, (Bard, P.Y., 2005). Las medidas se realizaron con un equipo CityShark, formado 
por un digitalizador de la casa Leas y un sismómetro triaxial Lennartz de 5s. La longitud de los registros fue de 15 minutos, y de 20 minutos en los emplazamientos donde se preveía mayor espesor de sedimentos. Se trabajó con ganancias de 512 y 1024 e intervalos de muestreo de $100 \mathrm{~Hz}$ Para calcular el cociente espectral H/V se ha utilizado el programa Geopsy, (Wathelet, 2003). El cálculo de los módulos de los espectros de Fourier se ha realizado dividiendo el registro en ventanas de 60 segundos y solapándolas un $50 \%$ entre ellas. Los espectros de Fourier se han suavizado con una función Konno \& Ohmachi. Los intervalos con ruido no estacionario se han eliminado aplicando un algoritmo anti-trigger STA/LTA.

En general, las medidas puntuales de ruido sísmico se distribuyeron en perfiles transversales a la estructura geológica y en emplazamientos cercanos a edificios dañados y esenciales. En la figura 4 se muestran dos fotografías tomadas durante la medida de ruido sísmico en dos emplazamientos de la ciudad de Lorca.
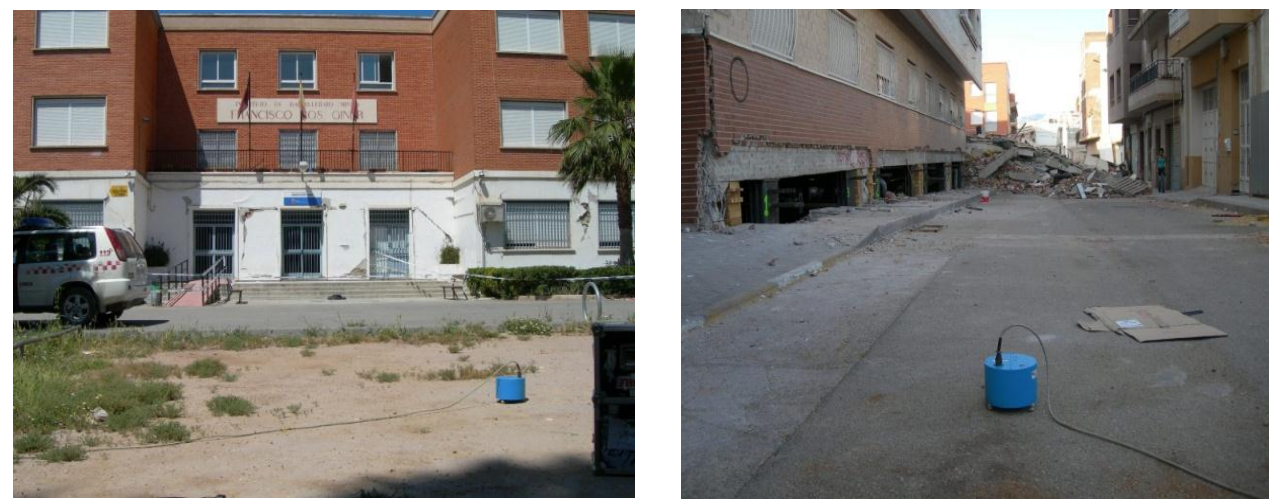

Fig. 4. Fotografías de dos emplazamientos de medida de ruido sísmico: punto LOR2 instituto Francisco Ros Giner (izquierda) y punto LOR7 edificio colapsado durante el sismo (derecha).

En algunos emplazamientos medidos se obtienen valores bajos de la frecuencia fundamental del suelo, entre 0.5 y $1.0 \mathrm{~Hz}$, que corresponderían a depósitos cuaternarios del valle del Guadalentín unos 100-200 metros de espesor identificados en un estudio del ITGE (1992) y en Martínez et al., (2012).

En general se observan valores bajos de amplitud en los gráficos $\mathrm{H} / \mathrm{V}$ y mucha dispersión, que pueden indicar la ausencia de contrastes mecánicos importantes entre suelo y roca. La presencia de varios picos se asocia a heterogeneidades verticales del terreno. Los emplazamientos sobre roca o con valores elevados de la frecuencia fundamental $(>10 \mathrm{~Hz})$ se encuentran al noroeste de la ciudad, cerca de los afloramientos rocosos. En la figura 5 se presenta, a modo de ejemplo, el cociente espectral H/V obtenido en dos emplazamientos de la ciudad (LOR15 y LOR09). 
Los resultados de la técnica del cociente espectral $\mathrm{H} / \mathrm{V}$ detectan un posible efecto de suelo en la ciudad de Lorca, pero no sería muy destacado. En algunos estudios realizados en otras zonas se han obtenido también relaciones espectrales con un comportamiento similar a las de Lorca, poca amplificación, dispersión y picos poco claros, de manera que el método no se ha podido utilizar para detectar posibles amplificaciones que, en cambio, se han confirmado comparando registros sísmicos en suelo y en roca así como a partir de la modelización de la respuesta sísmica del suelo utilizando perfiles de Vs obtenidos con técnicas sísmicas activas y pasivas de ondas superficiales. (Bonnefoy-Claudet et al., 2007; Belvaux et al., 2012).

En el caso de Lorca, el método $\mathrm{H} / \mathrm{V}$ no es concluyente porqué la ciudad se encuentra prácticamente sobre la zona de ruptura, por tanto, es posible que estemos en presencia de una configuración geológica y una geometría complejas, y que, además, los contrastes mecánicos entre los materiales no sean suficientes para poder identificar frecuencias fundamentales claras en algunos emplazamientos.
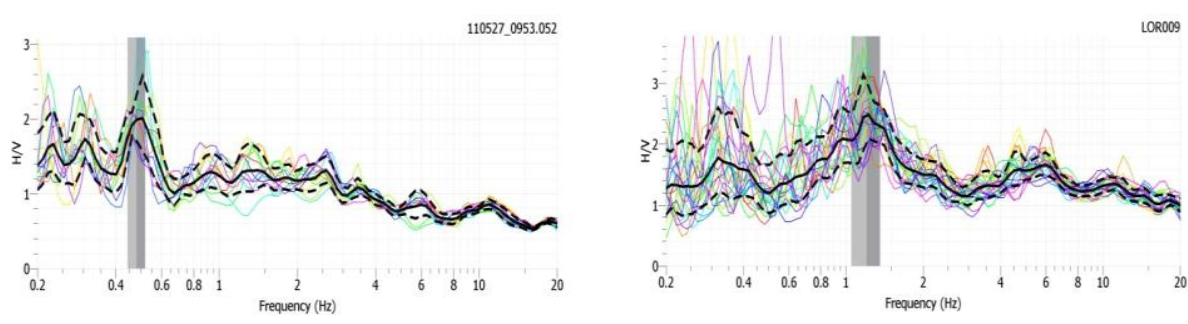

Fig. 5. Cocientes espectrales $\mathrm{H} / \mathrm{V}$ obtenidos en los emplazamientos LOR $15 \mathrm{f}=0,5 \mathrm{~Hz}$ (izquierda) y LOR09 $\mathrm{f}=1,2 \mathrm{~Hz}$ (derecha). En color se representan los cocientes espectrales para cada ventana de cálculo, y en negro la media de todas las ventanas junto con sus bandas de error (en discontinuo). Las franjas verticales de color gris identifican la frecuencia fundamental y su error.

\subsection{Clasificación de suelos según el Eurocódigo-8}

El Eurocódigo-8 (EC8), (CEN, 2004) propone una clasificación de suelos en función de rangos de valores promedio de la velocidad de propagación de las ondas de cizalla (Vs) en los 30 primeros metros de suelo $\left(\mathrm{Vs}_{30}\right)$.

En la ciudad de Lorca se realizaron medidas de ruido sísmico con la técnica de array (Aki, 1957, Wathelet, M. et al. 2004) en tres emplazamientos con el objetivo de obtener el perfil vertical de la velocidad de propagación de las ondas de cizalla, clasificarlos según el EC8 y definir una columna de suelo para modelizar su respuesta sísmica. Los emplazamientos seleccionados se ubican en suelo blando y cerca de zonas dañadas: recinto ferial, plaza de toros y centro médico.

La instrumentación utilizada consiste en un sismómetro Summit DMT de 24 canales y 11 geófonos Mark L4C 1Hz de componente vertical (Figura 6). En cada 
emplazamiento los geófonos se instalaron formando dos círculos concéntricos alrededor de un geófono central, el radio exterior tuvo valores entre 20 y 30 metros dependiendo del emplazamiento, siendo los radios interiores la mitad del exterior. La longitud del registro de ruido sísmico fue de 16 minutos con un intervalo de muestreo de $8 \mathrm{~ms}$. Los registros de cada array se han analizado con los métodos frecuencia-número de onda (FK) y autocorrelación espacial (SPAC) (Aki, 1957). El procesado de los registros de los array se ha realizado con el programa Geopsy (Wathelet, 2003). Además, en diferentes puntos de los arrays se calculó la frecuencia fundamental del suelo con la técnica del cociente espectral $\mathrm{H} / \mathrm{V}$ con el objetivo de validar la hipótesis de capas de suelos subhorizontales.
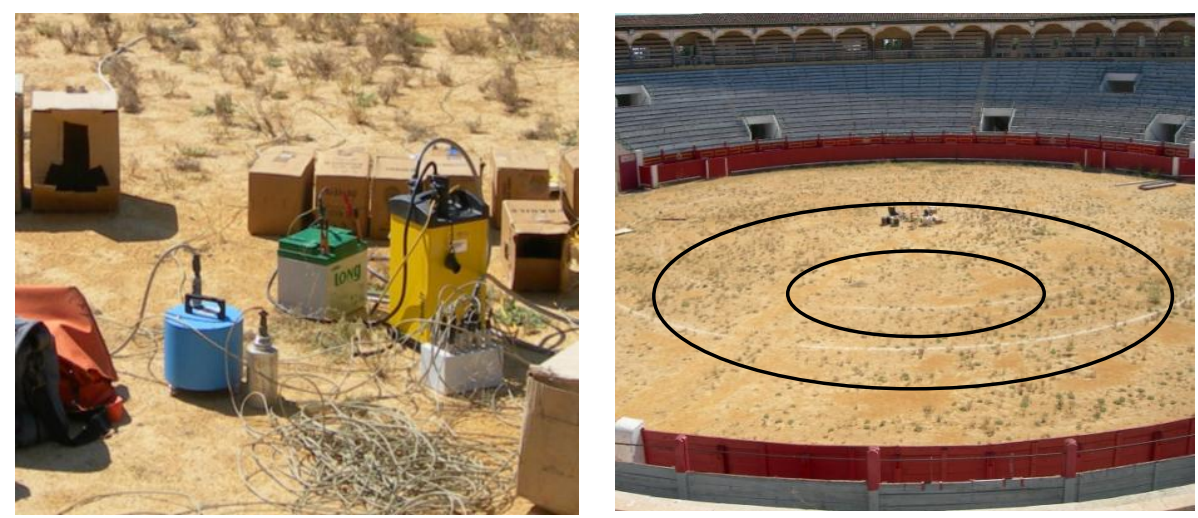

Fig. 6. Izda: Instrumentación utilizada en la medida de ruido sísmico con la técnica de array: sismómetro Summit DMT de 24 canales y geófono Mark L4C 1Hz de componente vertical. Dcha: Disposición de los sensores utilizada en las medidas con la técnica de array realizadas en Lorca. La longitud del radio de los círculos depende de las dimensiones de cada emplazamiento.

En la figura 7 se presentan los modelos del perfil vertical de la velocidad de propagación de las ondas de cizalla obtenidos en los emplazamiento estudiados. Estos modelos se han obtenido invirtiendo la curva de dispersión calculada con el método FK (Wathelet, 2003; Wathelet et al., 2004). En cada modelo, las curvas de color rojo son las que presentan menor error ("misfit"). Se muestran también los valores de la velocidad media de propagación de las ondas de cizalla calculados para un grosor de suelo de $30 \mathrm{~m}\left(\mathrm{Vs}_{30}\right)$ suponiendo que la última capa llega, como mínimo, a los 30 metros de profundidad. Y finalmente se representa la clasificación del tipo de suelo según la definición del EC8.

En los tres emplazamientos de los array realizados en Lorca se obtienen capas de suelo superficiales con Vs que corresponden aproximadamente a clases de suelo C del Eurocódigo (con valores de Vs comprendidos entre 180 y $360 \mathrm{~m} / \mathrm{s}$ ). A pesar de no apoyarse directamente sobre la roca, estas capas presentan contrastes mecánicos importantes por tanto, pueden amplificar las ondas sísmicas, como se puede ver en el apartado 4 de este artículo. 


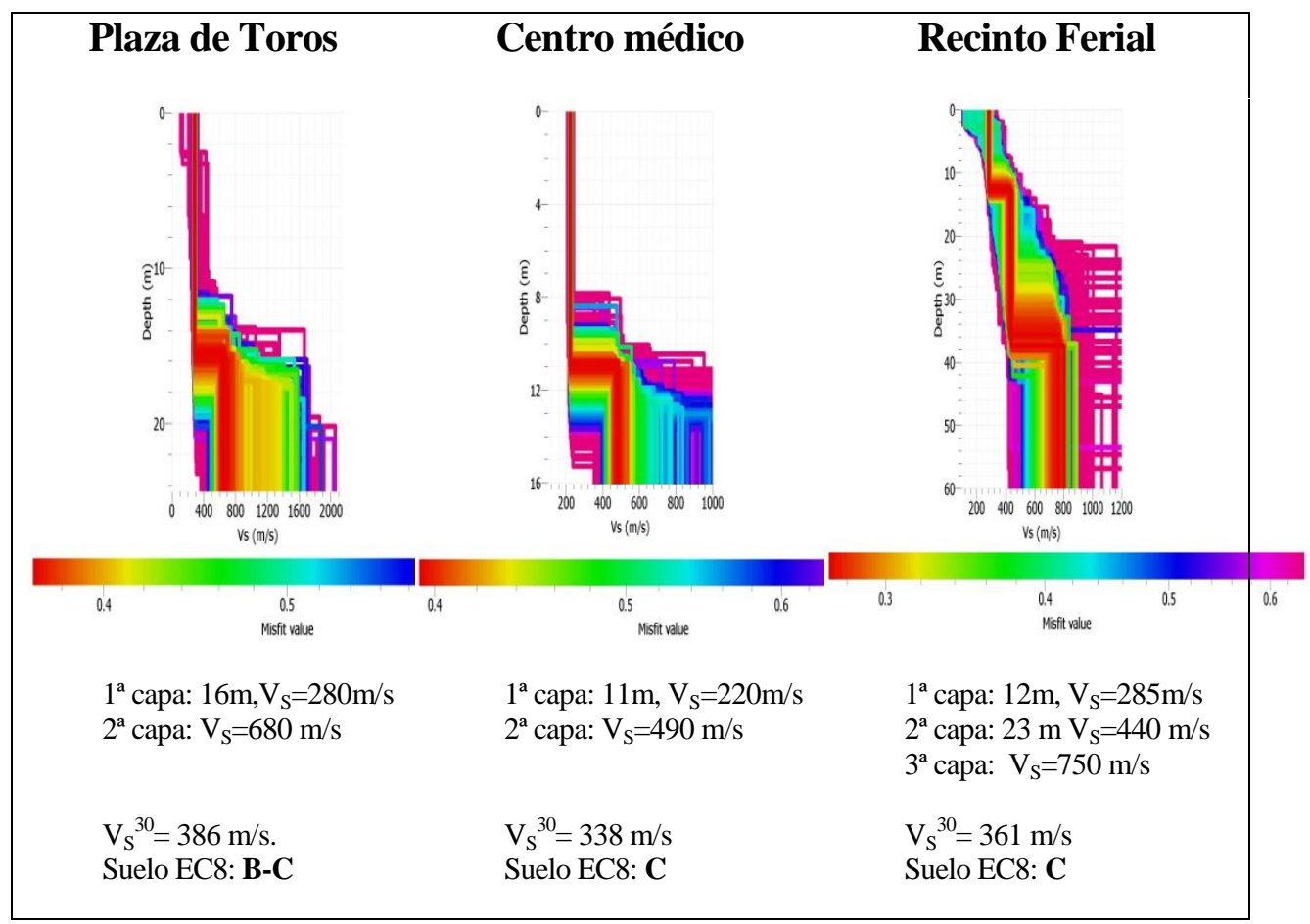

Fig. 7. Modelos del perfil vertical de velocidad de propagación de las ondas de cizalla (Vs) resultantes en la inversión de las curvas de dispersión obtenidas para los tres emplazamientos donde se realizaron medidas de ruido sísmico en array. En cada modelo, las curvas de color rojo son las que presentan menor error ("misfit"). Para cada emplazamiento se muestran los valores de la velocidad media de propagación de las ondas de cizalla para los primeros $30 \mathrm{~m}$ de suelo $\left(\mathrm{Vs}_{30}\right)$ y la clasificación del suelo según el EC8.

En la figura 8 se muestra, para cada uno de los tres emplazamientos, la comparación entre la curva de dispersión experimental calculada aplicando el método FK y las curvas de dispersión de los modelos teóricos obtenidos mediante el proceso de inversión. En general se observa un buen ajuste entre ellas, ya que en un amplio rango de frecuencias las curvas de dispersión experimentales se ajustan a las curvas de dispersión de los modelos teóricos con menor error. 


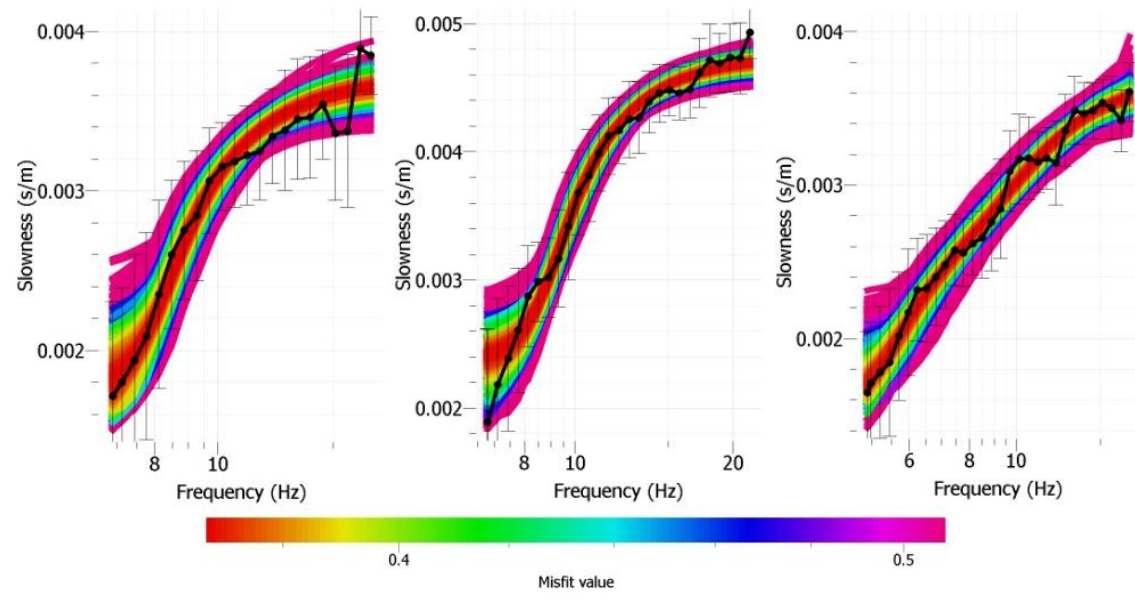

Fig. 8. Comparación entre las curvas de dispersión experimentales calculadas aplicando el método FK (negro) y las curvas de dispersión de los modelos teóricos obtenidos mediante la inversión (código de color) para cada unos de los array; izquierda: plaza de toros, centro: centro médico y derecha: recinto ferial.

\subsection{Red de intervención para el registro de réplicas.}

A partir del registro simultáneo de un sismo en estaciones instaladas en emplazamientos distintos, se puede calcular una función de transferencia experimental que permite caracterizar la respuesta sísmica de la capa de suelos de cada emplazamiento aplicando el método de las relaciones espectrales emplazamiento/referencia.

Con este objetivo, se instalaron tres estaciones sísmicas para registrar posibles réplicas del sismo principal en distintos tipos de suelo presentes en la ciudad de Lorca, (Figura 9). En la Tabla 1 se indican las coordenadas geográficas de los puntos donde se emplazaron estas estaciones, junto con la fecha y hora de instalación y retirada de estos equipos. Las estaciones sísmicas de la red de intervención estaban compuestas por un sismómetro triaxial Lennartz de 5 segundos y un digitalizador Spider de Worldsensing. Se trabajó en registro continuo y con un intervalo de muestreo de $100 \mathrm{~Hz}$.

Tabla. 1. Coordenadas geográficas ED50, fecha y hora de la puesta en marcha y retirada de las estaciones de la red de intervención instalada en la ciudad de Lorca

\begin{tabular}{|c|c|c|c|c|c|}
\hline Estación & Latitud & Longitud & Inicio & Fin & Suelo \\
\hline Ayuntamiento & $37^{\circ} 40^{\prime} 39.9^{\prime} \mathrm{N}$ & $1^{\circ} 41^{\prime} 54.8^{\prime} \mathrm{W}$ & $24 / 0518: 00$ & $27 / 0513: 30$ & Suelo duro \\
\hline Escuela P. Hita & $37^{\circ} 39^{\prime} 48.8^{\prime} \mathrm{N}$ & $1^{\circ} 42^{\prime} 35.0^{\prime} \mathrm{W}$ & $24 / 0519: 00$ & $27 / 0513: 00$ & Roca \\
\hline Centro médico & $37^{\circ} 39^{\prime} 52.0^{\prime} \mathrm{N}$ & $1^{\circ} 41^{\prime} 42.5^{\prime} \mathrm{W}$ & $25 / 0509: 00$ & $27 / 0512: 30$ & Suelo blando \\
\hline
\end{tabular}



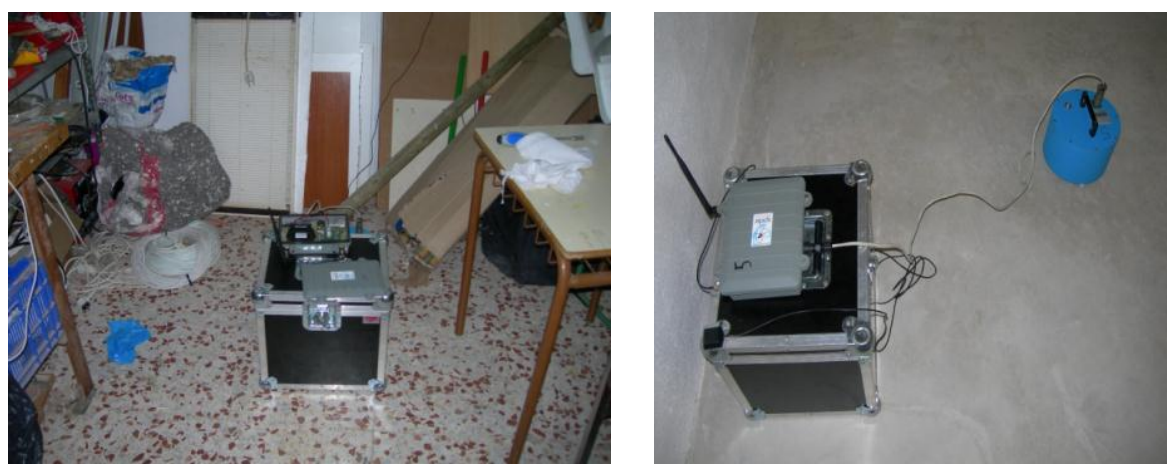

Fig. 9. Emplazamientos de las estaciones de la red de intervención en Lorca: Escuela Pérez de Hita (izquierda) y Centro médico (derecha).

Durante los días de funcionamiento la red de intervención solo se produjo una réplica (Tabla 2), ésta fue registrada por las dos estaciones que estaban instaladas en el momento del sismo (Ayuntamiento y Escuela Pérez de Hita). Además también se pudo disponer del sismograma de la réplica registrado en la unidad móvil que el IGN instaló en Lorca (UMV6).

En la Figura 10 se presenta un mapa con la ubicación de las estaciones sísmicas y del epicentro así como una comparación del sismograma obtenido en la Escuela Pérez de Hita (roca, A) con el obtenido en la estación móvil del IGN (suelo blando, C). El movimiento del suelo registrado en la estación del IGN, emplazada en suelo tipo $\mathrm{C}$, tiene una amplitud superior (factor 2) al registrado en la estación en roca (escuela).

Tabla 2. Fecha/hora, coordenadas geográficas ETRS-89 y magnitud de la réplica registrada por la red de intervención instalada en la ciudad de Lorca.

\begin{tabular}{|l|l|l|c|c|}
\hline Fecha & Hora & Latitud & Longitud & Mag (mbLg) \\
\hline 24/05/2011 & $23: 10: 56$ & $37,7089 \mathrm{~N}$ & $1,6475 \mathrm{~W}$ & 1,8 \\
\hline
\end{tabular}

En la figura 11 se comparan los espectros de respuesta en pseudovelocidad calculados a partir del acelerograma registrado en la estación móvil del IGC (suelo C) y en la Escuela Pérez de Hita (suelo A), en ambas componentes se observa una amplificación general del espectro en suelo respecto al de roca en un factor 2.

Debido a que la distancia entre las estaciones sísmicas $(<2 \mathrm{~km})$ es del mismo orden de magnitud que la distancia epicentral $(<7 \mathrm{~km})$, no se ha calculado la función de transferencia de la capa de suelos aplicando el método de las relaciones espectrales empíricas suelo/roca porque el término de trayectoria puede que no sea el mismo para las dos estaciones. 

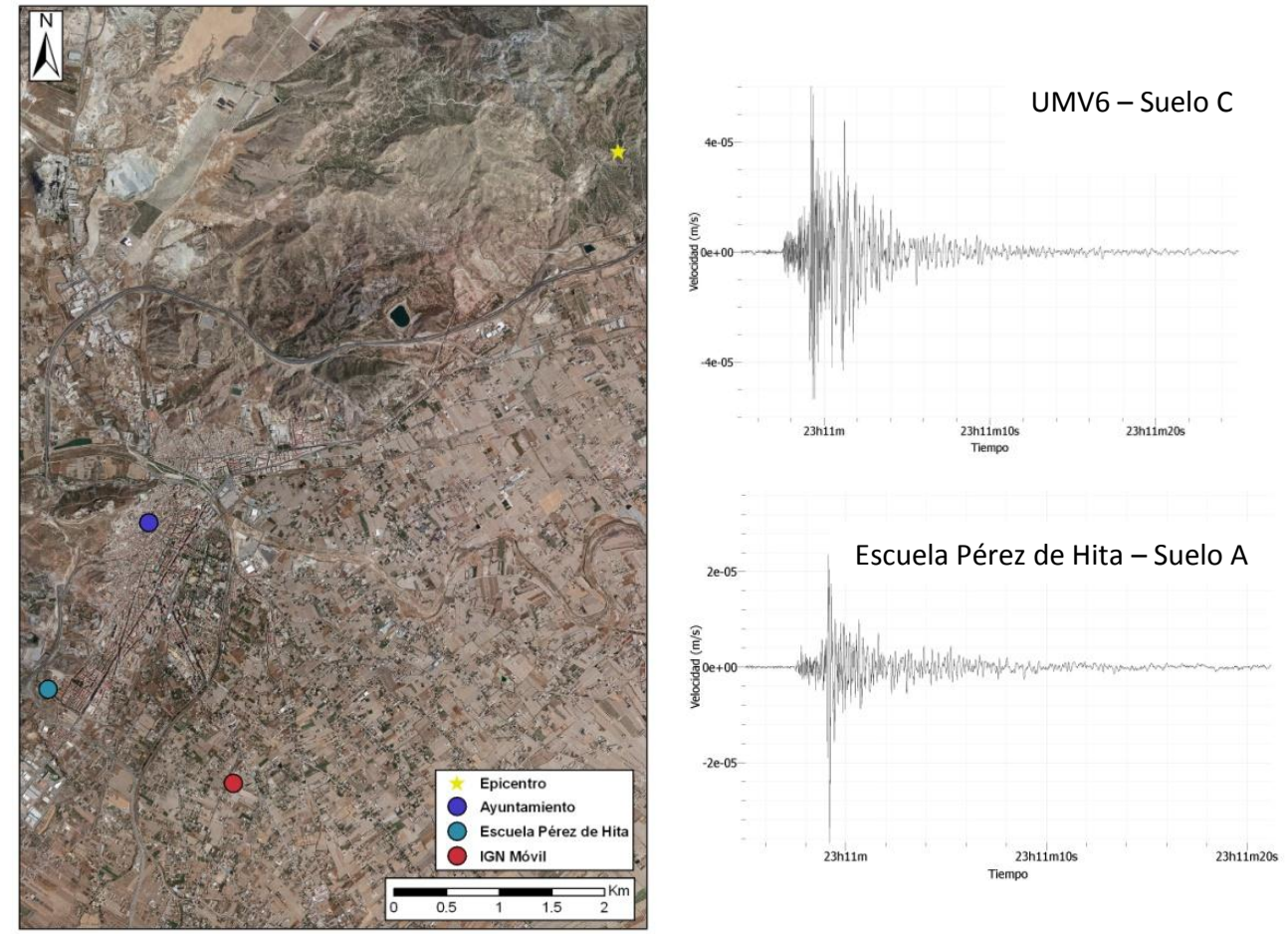

Fig. 10. Izda.: Mapa de situación de las estaciones de intervención y del epicentro. Dcha.: comparación entre dos registros de la réplica del día 24/05/201 obtenidos en la estación móvil del IGN (UMV6) instalada en suelo (arriba) y la estación instalada en roca, en la Escuela Pérez de Hita, (abajo).

\section{Modelización de la respuesta sísmica del suelo}

Se ha modelizado la respuesta sísmica en los emplazamientos donde se realizaron medidas en array, para ello se ha aplicado el método 1D lineal equivalente (Proshake). Se han definido columnas representativas de la capa de suelo a partir de los perfiles de Vs y de información geológica previa (Tabla 3).

Como sismo de entrada en roca se ha considerado el registro del sismo principal de $\mathrm{Mw}=5.1$ obtenido en la estación acelerométrica que el IGN tiene instalada en el ayuntamiento de Lorca, es un suelo tipo B y por tanto este es el suelo de referencia que se ha utilizado. 
Comp NS

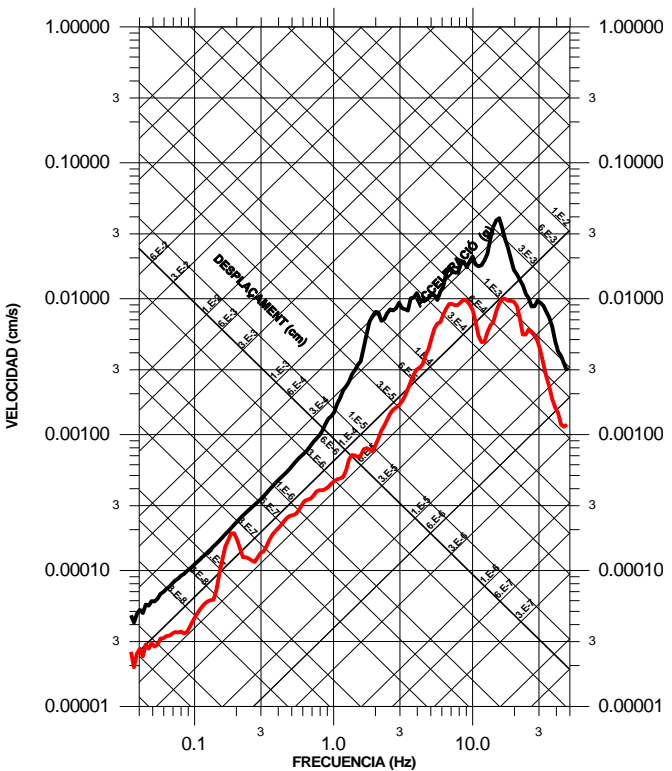

- Unidad Móvil

Escuela Pérez Hita
Comp EW

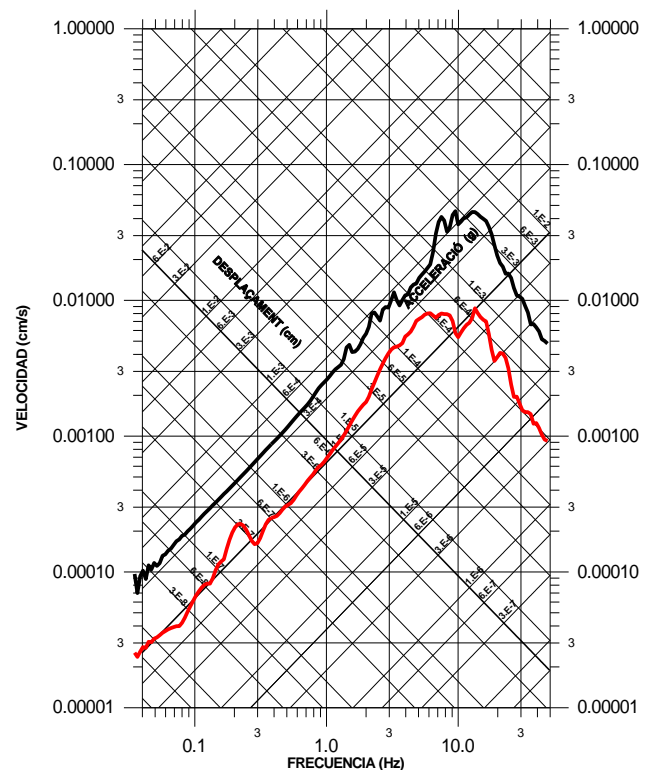

— Unidad Móvil

Fig. 11. Comparación de los espectros PSV obtenidos a partir de los registros de la réplica de magnitud 1.8 en roca (rojo) y en suelo blando (negro). Izda.: componente NS. Dcha.: componente EW.

Como resultado de la modelización se han obtenido, para cada columna de suelo, las funciones de transferencia y los registros sintéticos de aceleración en suelo, para cada uno de ellos se han calculado los espectros de respuesta en aceleraciones y las intensidades de Arias (AI) (Arias, 1970). A partir de la relación empírica entre Intensidad de Arias e intensidad macrosísmica propuesta por Cabañas et al. (1997) para el área Mediterránea se ha estimado incremento de intensidad macrosísmica respecto a la intensidad en roca. 
Tabla 3. Parámetros geológicos y geotécnicos de las columnas de suelo utilizadas en la modelización de la respuesta sísmica del suelo.

ARRAY 1. Plaza de toros

\begin{tabular}{|c|c|c|c|c|c|c|c|}
\hline Capa & $\begin{array}{c}\text { Tipo de } \\
\text { material }\end{array}$ & $\begin{array}{c}\text { Grosor } \\
(\mathbf{m})\end{array}$ & $\begin{array}{c}\text { Peso } \\
\text { unitario } \\
\left(\mathbf{K N} / \mathbf{m}^{3}\right)\end{array}$ & $\begin{array}{c}\text { Gmax } \\
(\mathbf{M P a})\end{array}$ & $\begin{array}{c}\text { Vs } \\
(\mathbf{m} / \mathbf{s})\end{array}$ & Curva módulo G & $\begin{array}{c}\text { Curva } \\
\text { amortiguamiento }\end{array}$ \\
\hline 1 & $\begin{array}{c}\text { Limo } \\
\text { arcilloso }\end{array}$ & 16.0 & 17.2 & 137.1 & 280 & $\begin{array}{c}\text { Arcilla (Seed and } \\
\text { Sun, 1989) }\end{array}$ & $\begin{array}{c}\text { Arcilla (Idriss, } \\
1990)\end{array}$ \\
\hline 2 & Neógeno & $\infty$ & 22.0 & 1037.3 & 680 & Roca & Roca \\
\hline
\end{tabular}

ARRAY 2. Centro médico

\begin{tabular}{|c|c|c|c|c|c|c|c|}
\hline Capa & $\begin{array}{c}\text { Tipo de } \\
\text { material }\end{array}$ & $\begin{array}{c}\text { Grosor } \\
(\mathbf{m})\end{array}$ & $\begin{array}{c}\text { Peso } \\
\text { unitario } \\
\left(\mathbf{K N} / \mathbf{m}^{3}\right)\end{array}$ & $\begin{array}{c}\text { Gmax } \\
(\mathbf{M P a})\end{array}$ & $\begin{array}{c}\text { Vs } \\
(\mathbf{m} / \mathbf{s})\end{array}$ & $\begin{array}{c}\text { Curva módulo } \\
\mathbf{G}\end{array}$ & $\begin{array}{c}\text { Curva } \\
\text { amortiguamiento }\end{array}$ \\
\hline 1 & Limos & 11.0 & 17.2 & 84.6 & 220 & $\begin{array}{c}\text { Arcilla (Seed } \\
\text { and Sun, 1989) }\end{array}$ & $\begin{array}{c}\text { Arcilla (Idriss, } \\
1990)\end{array}$ \\
\hline 2 & Neógeno & $\infty$ & 22.0 & 538.6 & 490 & Roca & Roca \\
\hline
\end{tabular}

ARRAY 3. Recinto Ferial

\begin{tabular}{|c|c|c|c|c|c|c|c|}
\hline Capa & $\begin{array}{c}\text { Tipo de } \\
\text { material }\end{array}$ & $\begin{array}{c}\text { Grosor } \\
(\mathbf{m})\end{array}$ & $\begin{array}{c}\text { Peso } \\
\text { unitario } \\
\left(\mathbf{K N} / \mathbf{m}^{\mathbf{3}}\right)\end{array}$ & $\begin{array}{c}\mathbf{G m a x} \\
\mathbf{( M P a})\end{array}$ & $\begin{array}{c}\mathbf{V s} \\
\mathbf{( m / s})\end{array}$ & $\begin{array}{c}\text { Curva módulo } \\
\mathbf{G}\end{array}$ & $\begin{array}{c}\text { Curva } \\
\text { amortiguamiento }\end{array}$ \\
\hline 1 & Limos & 12.00 & 17.15 & 142.1 & 285 & $\begin{array}{c}\text { Arcilla (Seed } \\
\text { and Sun, 1989) }\end{array}$ & $\begin{array}{c}\text { Arcilla (Idriss, } \\
1990)\end{array}$ \\
\hline 2 & Limos & 23.00 & 17.15 & 338.6 & 440 & $\begin{array}{c}\text { Arcilla (Seed } \\
\text { and Sun, 1989) }\end{array}$ & $\begin{array}{c}\text { Arcilla media (Sun } \\
\text { et al., 1988) }\end{array}$ \\
\hline 3 & Neógeno & $\infty$ & 22.00 & 1261.9 & 750 & Roca & Roca \\
\hline
\end{tabular}

En la figura 12 se presentan, para cada emplazamiento, las funciones de transferencia de la capa de suelos calculadas mediante simulación numérica, los espectros de respuesta en aceleración para suelo y roca, la clasificación del suelo según el EC8 y el incremento de intensidad macrosísmica respecto a la intensidad en roca calculado a partir de los registro del movimiento del suelo modelizado.

La frecuencia fundamental de las funciones de transferencia en los tres emplazamientos se encuentra entre 1 y $3 \mathrm{~Hz}$. Se observan amplificaciones en los espectros de aceleración en suelo (SA) respecto al emplazamiento de referencia (suelo B). La función de transferencia del recinto ferial (suelo C) muestra la contribución de varias capas de suelo, la frecuencia fundamental se obtiene a $2 \mathrm{~Hz}$, En este emplazamiento se ha estimado un incremento de intensidad macrosísmica de 1 grado respecto a la intensidad en roca 


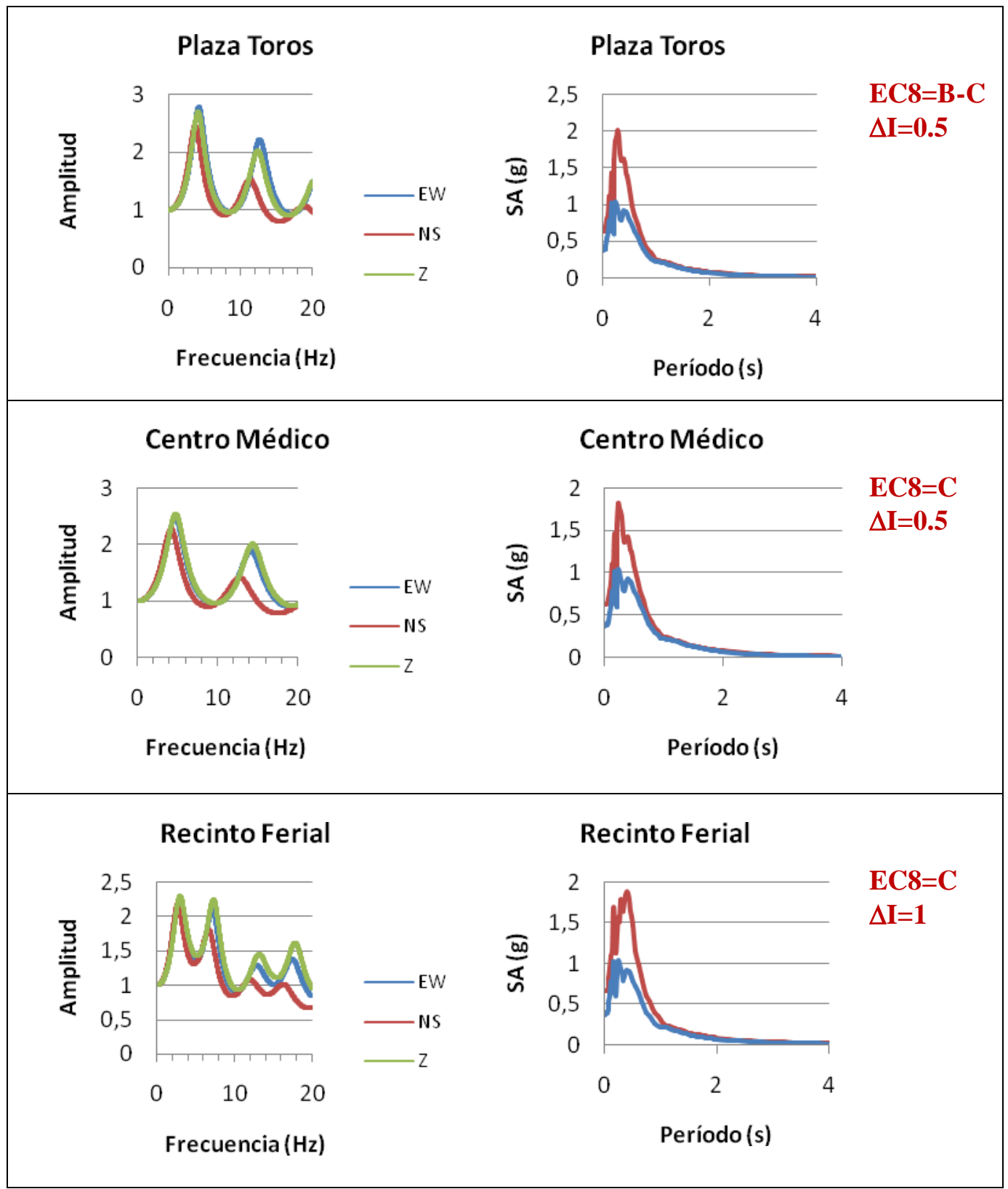

Fig. 12. Resultados obtenidos en la modelización de la respuesta sísmica de la capa de suelo en tres emplazamientos a partir del registro en roca (suelo B) del sismo principal: Funciones de transferencia modelizadas, comparación entre los espectros de respuesta en aceleración (SA) de los registros en suelo (rojo) y roca (azul), clasificación del tipo de suelo según el EC8 e incremento de intensidad macrosísmica debido al efecto de suelo. 


\section{Conclusiones}

A partir de una veintena de medidas de ruido sísmico ambiental, los valores de la frecuencia fundamental del suelo obtenidos en Lorca parecen bajos, la mayoría de ellos varía entre 0,5 y $1,0 \mathrm{~Hz}$, con algunos emplazamientos a $10 \mathrm{~Hz}$ y otros entre 4$5 \mathrm{~Hz}$. Estas frecuencias corresponden a depósitos de suelos con grosores de centenares de metros.

Con la técnica del cociente espectral $\mathrm{H} / \mathrm{V}$ no se han observado contrastes importantes entre suelo y roca, en este caso, el método no ha resultado ser un buen indicativo para detectar efectos de amplificación.

Los resultados obtenidos con la técnica de array nos indican la presencia de capas superficiales de suelos con Vs bajas (clases de suelo C del Eurocódigo EC8) que podrían haber amplificado las ondas sísmicas durante el sismo de Lorca.

Los perfiles de Vs obtenidos con la técnica de arrays han permitido definir columnas de suelo representativas de cada emplazamiento y se ha modelizado la respuesta sísmica del suelo considerando como sismo de entrada en roca el registro del sismo principal. De esta manera se ha podido estimar el incremento de intensidad macrosísmica debido al efecto de suelo obteniéndose valores de 0.5 en suelos tipo B-C (EC8), II-III (NCSE) y valores de 1 en suelos tipo C (EC8), III (NCSE).

A partir del registro de réplicas se ha comprobado que en los emplazamientos situados sobre suelo blando se amplificó el movimiento del suelo en un factor 2 .

Las dos conclusiones anteriores podrían indicar que el valor de intensidad VII estimado por el IGN como valor promedio para toda la ciudad, podría haber variado localmente entre VI-VII para emplazamientos rocosos y VII-VIII para emplazamientos en suelos de tipo C.

Los efectos de suelo pueden haber contribuido al aumento de daños en los edificios del valle del Guadalentín.

\section{Agradecimientos}

Agradecemos la colaboración de las personas del IGN (Instituto Geográfico Nacional) y de la AFPS (Asociación Francesa de Ingeniería Sísmica) que han participado en la realización de este trabajo. En particular la colaboración prestada por la Policía Municipal de la ciudad de Lorca durante los tres días en que se realizaron medidas en el área urbana de dicha localidad.

\section{Referencias bibliográficas}

AFPS (2011). Le seisme de Lorca (Espagne) du 11 mai 2011. Rapport de mission de l'Association Française de Génie Parasismique, 151p.

AKI, K. (1957). Space and time spectra of stationary stochastic waves, with special reference to microtremors. Bull. Earth. Res. Inst. 35, 415-456.

AKI, K. (1993). Local site effects on weak and strong ground motion. Tectonophysics, 218, 93-111. 
ARIAS, A. (1970). A measure of earthquake intensity, in R.J. Hansen, ed. Seismic Design for Nuclear Power Plants. MIT Press, Cambridge, Massachusetts, 438483.

BARD, P.Y. (1985). Les effets de site d'origine structurale : principaux résultats expérimentaux et théoriques. A : Genie Parasismique. Ed: Victor Davidovici. Paris, pp 223-238.

BARD, P.Y \& the SESAME team (2005). SESAME European research project (WP12-Deliverable D23.12). BARD, P.Y. (coord.). Guidelines for the implementation of the $\mathrm{H} / \mathrm{V}$ spectral ratio technique on ambient vibration measurements, processing and interpretation. 62p. Available at http://sesamefp5.obs.ujf-grenoble.fr.

BENITO, B., MURPHY, P., TSIGE, M., MARTÍNEZ DÍAZ, J.J., GASPARESCRIBANO, J.M., GARCÍA-MAYORDOMO, J., JIMENÉZ, M.E., GARCÍA, M.J., CANORA, C., ÁlVAREZ-GÓMEZ, J. A. y GARCÍA-FLORES, I. (2006). Riesgo Sísmico en la Región de Murcia: Proyecto RISMUR. Informe final, 7 volúmenes y un anexo, Instituto Geográfico Nacional. Protección Civil Región de Murcia.

BELVAUX, M., ROULlÉ, A., AUClAIR, S.,VANOUDHEUSDEN, E. \& BARRAS, A.-V. (2012). Limites des methodes de caracterisation des effets de site dans les microzonages sismiques. Journées Nationales de Géotechnique et de Géologie de l'Ingenieur JNGG2012 - Bordeaux 4-6 juillet.

BONNEFOY-CLAUDET S., BAIZE, S.,BONOLLA, F., BERGE-THIERRY, C., CAMPOS, J., PASTEN, C., VERDUGO, J. \& VOLANT, PH. (2007). Comment caracteriser les effets de site des structures complexesà partir du bruit ambiant. Application au bassin de Santiago du Chili. $7^{\text {ème }}$. Colloque National AFPS, Ecole Centrale Paris, France, 4-6 juillet.

CABAÑAS, L., BENITO, B., \& HERRAIZ, M. (1997). An approach to the measurement of the potential structural damage of earthquake ground motion. Earthquake Engineering and Structural Dynamics, 26, 79-92.

CEN (2004), Comité Européen de Normalisation, Eurocode-8: Design of structures for earthquake resistance. Part 1, Doc CEN/TC250/SC8/N335, January. Bruselas, 229 pp.

DIRECCIÓN GENERAL DE PROTECCIÓN CIVIL (DGPC), (2006). Plan especial de riesgo sísmico de la región de Murcia. SISMIMUR. Consejería de Presidencia, Comunidad Autónoma de la región de Murcia.

FIGUERAS, S \& MACAU A., (2009). Efectos locales y microzonificación sísmica. ¿Se pueden producir daños lejos de la zona epicentral? En: Terremotos. Cuando la Tierra Tiembla. A. Ugalde (coord.). Colección divulgación CSIC. Ed. Catarata. Madrid. 135-144.

ISDRISS, I.M.(1990). Response of soft soil sites during earthquakes. Proc. H. Bolton Seed Memorial Symposium, J.M. Duncan (editor), 2, 273-290. 
IGC, (2011). El Terremoto de Lorca del 11 de mayo de 2011. Informe de la inspección y de los trabajos de campo realizados. IGC, UPC, AEIS. Monografía técnica $n^{\circ}$ 3. Barcelona. $86 \mathrm{pp}$.

IGME, (2011). Informe geológico preliminar del terremoto de Lorca del 11 de mayo del año 2011, 5.1 Mw. Instituto Geológico y Minero de España (IGME), Grupo de Tectónica Activa, Paleosismicidad y Riesgos Asociados de la Universidad Complutense de Madrid (UCM), Universidad Autónoma de Madrid (UAM) y la Universidad Rey Juan Carlos de Madrid (URJC)

IGN, (2011).Serie terremoto NE Lorca, 11 mayo 2011. http://www.ign.es/ ign/resources/sismologia/Lorca.pdf

ITGE, (1992). Estudio de peligrosidad y vulnerabilidad sísmica en Lorca y su término municipal Instituto Tecnológico y Geominero de España (ITGE), Empresa de Estudios Geológicos- Geotécnicos (GEONOC).

JIMÉNEZ, M. J. \& GARCÍA FERNÁNDEZ, M., (2009). Peligrosidad sísmica. Qué efectos podemos esperar de los terremotos, dónde, cuándo y de qué tamaño. En: Terremotos. Cuando la Tierra Tiembla. A. Ugalde (coord.). Colección divulgación CSIC. Ed. Catarata. Madrid. 123-134.

MACAU, A. (2008). Microzonación sísmica. Contribución a los estudios de peligrosidad sísmica a escala local en zonas rurales y urbanas. Tesis Doctoral. Universidad Politécnica de Catalunya. Barcelona, 328 pp.

MARTÍNEZ-DÍAZ, J.J., BEJAR-PIZARRO, M., ÁLVAREZ-GÓMEZ, J.A.,C, MANCILLA, F.L., STICH, D., HERRERA, G., \& MORALES, J. (2012). Tectonic and seismic implications of an intersegment rupture.The damaging May 11th $2011 \mathrm{Mw} 5.2$ Lorca, Spain, earthquake. Tectonophysics. (In press).

NAVARRO, M., GARCÍA-JEREZ, J.A., VIDAL, F., ENOMOTO, T., PÉREZRUIZ, J.A., ALCALÁ, F.J., LUZÓN, F., YAMAMMOTO, T. y IWATATE, T. (2006). Características del movimiento de suelo a partir de medidas de vibración ambiental y registros de aceleración. $5^{a}$ Asamblea Hispano-Portuguesa de Geodesia y Geofísica. Sevilla

NAVARRO, M., GARCÍA-JEREZ, JA., ALCALÉ, FJ., VIDAL, F., ENOMOTO, T., LUZÓN, F. \& CREUS, C. (2008). $\mathrm{V}_{\mathrm{S}}{ }^{30}$ Structure of Lorca town (SE Spain) from Ambient Noise Array Observations. $31^{s t}$ General Assembly of the European Seismoligical Commission ESC 2008. Hersonissos, Crete, Greece, 712 September.

NAVARRO, M., GARCÍA-JEREZ, A., ALCALÁ, F., VIDAL, F. \& ENOMOTO, T. (2012). Local site effect microzonation of Lorca town (southern Spain). Bull Earthquake Eng (under review).

SEED, H.B. \& SUN, J.H. (1989). Implication of site effects in the Mexico City earthquake of Septempber 19,1989 for earthquake-resistance-desing criteria in the San Francisco Bay Area of California. Report No. UCB/EERC - 89/03, University of California, Berkerley, California. 
SUN, J.I., GOLESORKHI, R. \& SEED, H.B. (1988). Dynamic moduli and damping ratios for cohesive soils. Report No. EERC - 88/15, Earthquake Engineering, ASCE, 112, 11,1016-1032.

WATHELET, M. (2003). Report on the inversion of velocity profile and version of the inversion software. SESAME report D14.07.

WATHELET, M., D. JONGMANS, D. \& M. OHRNBERGER, M.. (2004). Surface wave inversion using a direct search algorithm and its application to ambient vibration measurements. EGU, first general assembly, Nice, France. 